\title{
Gastric Ewing sarcoma/primitive neuroectodermal tumor: A case report
}

\author{
MAKOTO INOUE $^{1}$, TOSHIFUMI WAKAI ${ }^{1}$, PAVEL V. KORITA $^{2}$, JUN SAKATA ${ }^{1}$, \\ RYO KUROSAKI $^{1}$, AKIRA OGOSE ${ }^{3}$, HIROYUKI KAWASHIMA ${ }^{3}$, YOSHIO SHIRAI ${ }^{1}$, \\ YOICHI AJIOKA $^{2}$ and KATSUYOSHI HATAKEYAMA ${ }^{1}$ \\ Divisions of ${ }^{1}$ Digestive and General Surgery, ${ }^{2}$ Molecular and Diagnostic Pathology, and ${ }^{3}$ Orthopedic Surgery, \\ Niigata University Graduate School of Medical and Dental Sciences, Chuo-ku, Niigata 951-8510, Japan \\ Received October 25, 2010; Accepted January 13, 2011
}

DOI: $10.3892 / \mathrm{ol} .2011 .246$

\begin{abstract}
Ewing sarcoma/primitive neuroectodermal tumors (ES/PNETs) may arise in bone or soft tissue; however, these tumors rarely originate in the stomach. To the best of our knowledge, only four cases have previously been reported in the English-language literature. A 41-year-old Japanese woman was admitted with abdominal pain and underwent gastrectomy to remove the primary tumor. Immunohistochemistry, chromosomal karyotype and molecular analysis using reverse transcription-polymerase chain reaction were performed in the tumor specimens obtained. Tumor cells showed positive immunoreactivity for CD99, vimentin, CD117 (c-kit), S100, chromogranin A and synaptophysin. The tumor was a gastric ES/PNET with the EWS-FLII fusion gene translocation $\mathrm{t}(11 ; 22)$ (q24;q12). Multiple repeat metastasectomies, as well as multi-agent chemotherapy and radiotherapy were performed for recurrent disease. Despite treatment, the patient succumbed due to progressive disease 110 months after the initial surgery for gastric ES/PNET. A review of the reported cases suggests that patients with gastric ES/PNETs have an unfavorable prognosis following resection due to the high propensity of these tumors to metastasize. Thus, multimodal treatment approaches including surgery, as well as multi-agent chemotherapy and radiotherapy may provide a survival benefit for patients with gastric ES/PNETs.
\end{abstract}

Correspondence to: Dr Toshifumi Wakai, Division of Digestive and General Surgery, Niigata University Graduate School of Medical and Dental Sciences, 1-757 Asahimachi-dori, Chuo-ku, Niigata, 951-8510, Japan

E-mail: wakait@med.niigata-u.ac.jp

Abbreviations: ES, Ewing sarcoma; PNET, primitive neuroectodermal tumor; RECIST, Response Evaluation Criteria in Solid Tumors

Key words: gastric neoplasm, Ewing sarcoma, primitive neuroectodermal tumor, CD99

\section{Introduction}

Ewing sarcoma/primitive neuroectodermal tumors (ES/ PNETs) are malignant tumors whose cells typically express the CD99 surface antigen encoded by the MIC2 gene (1). ES/PNETs most commonly arise in bone, and occasionally originate in soft tissue (1). However, it is relatively rare for ES/PNETs to arise from the stomach. Only four cases of gastric ES/PNETs have been reported in the English-language literature (PubMed, National Library of Medicine, Bethesda, MD, USA) prior to September 2010 (2-5). In this study, the four cases were assessed and an additional case was reported to further characterize the clinicopathological parameters in gastric ES/PNETs.

\section{Case report}

A 41-year-old Japanese woman was admitted with abdominal pain in 2000 to Saiseikai Niigata Second Hospital, Niigata, Japan. A contrast-enhanced computed tomography scan revealed a solid mass in the anterior wall of the stomach (Fig. 1A). She underwent a distal gastrectomy and regional lymphadenectomy. The resected specimen contained a firm, round tumor with extraluminal growth, 90x55 mm in diameter, located in the anterior wall of the stomach (Fig. 1B). Histological examination revealed the tumor arising from the muscularis externa of the stomach (Fig. 1C). Tumor cells were characterized by uniform, compact, round to oval nuclei, a modest amount of pale eosinophilic cytoplasm, and pseudorosette formation (Fig. 1D). Tumor cells showed positive immunoreactivity for CD99 (Fig. 1D), vimentin, CD117 (c-kit), S100, chromogranin A and synaptophysin. The chromosomal karyotype demonstrated the translocation $\mathrm{t}(11 ; 22)(\mathrm{q} 24 ; \mathrm{q} 12)$ (Fig. 2). Molecular analysis using reverse transcriptionpolymerase chain reaction revealed the EWS-FLII fusion gene translocation $\mathrm{t}(11 ; 22)(\mathrm{q} 24 ; \mathrm{q} 12)$. Thus, the primary gastric tumor was determined to be an ES/PNET.

During the 6 years following the initial surgery, the patient underwent five metastasectomies for isolated peritoneal metastases. A follow-up computed tomography scan performed 6 years post-gastrectomy showed multiple intra-abdominal peritoneal metastases. The patient received three courses 


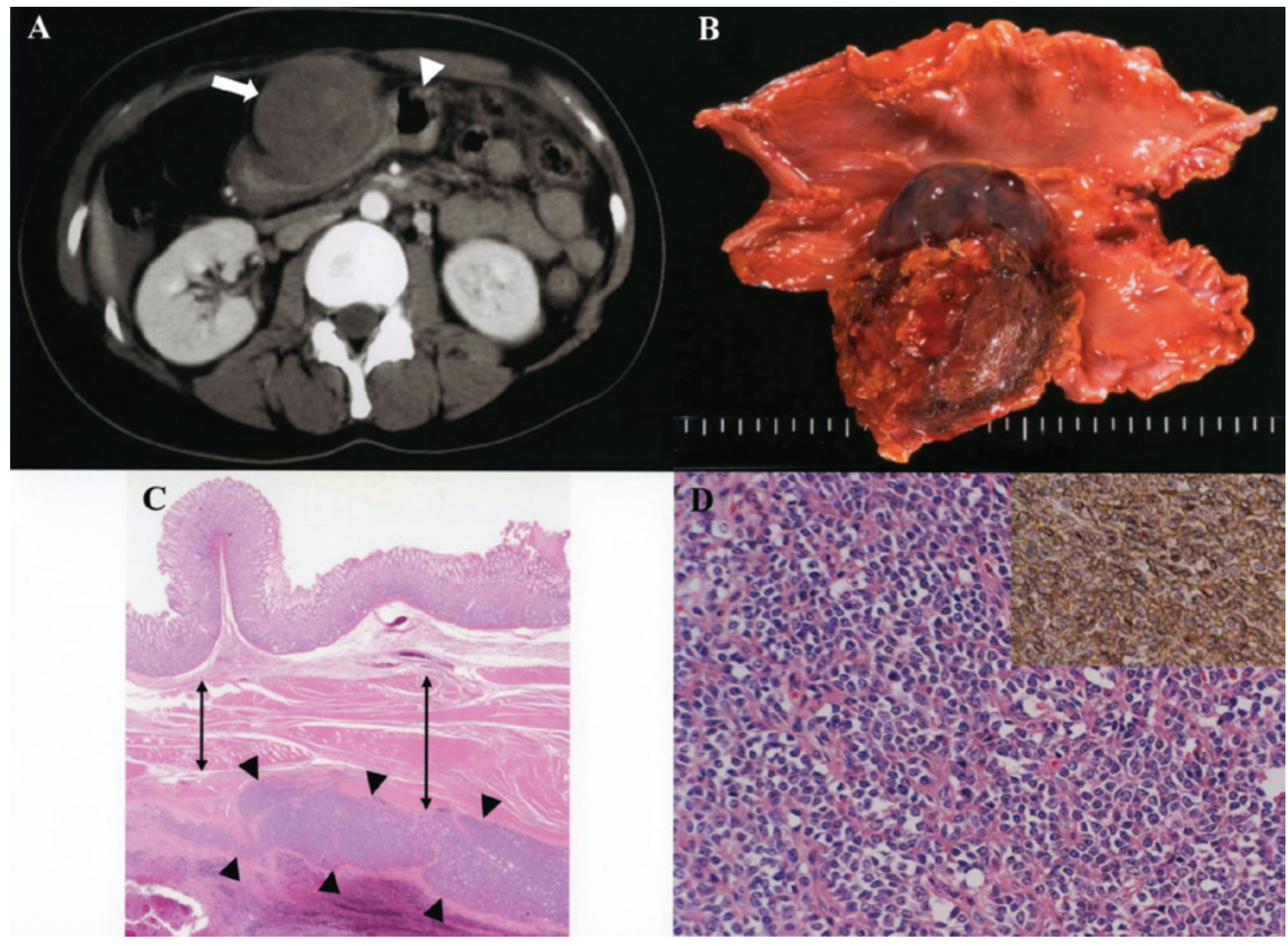

Figure 1. (A) Contrast-enhanced computed tomography image showing a solid mass (arrow) arising from the stomach (arrowhead). (B) The resected specimen showing the solid round tumor with extraluminal growth, located in the anterior wall of the stomach. (C) Hematoxylin and eosin staining. The tumor (arrowheads) with extraluminal growth expanding from the gastric muscularis externa (double headed arrows) is shown. (Original magnification, x10). (D) Hematoxylin and eosin staining. The tumor cells were characterized by uniform, compact, round to oval nuclei, a modest amount of pale eosinophilic cytoplasm and pseudorosette formation. Upper right inset indicates the positive immunoreactivity of tumor cells when stained with an anti-CD99 antibody. (Original magnification, $\mathrm{x} 400)$.

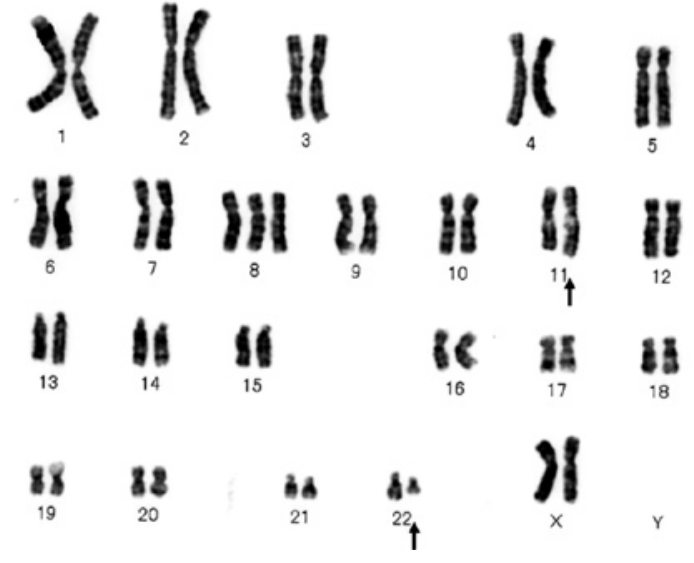

Figure 2. Karyotype showing translocation $\mathrm{t}(11 ; 22)(\mathrm{q} 24 ; \mathrm{q} 12)$ and presence of trisomy 8 . Arrows indicate chromosomes altered by the translocation.

of chemotherapy according to the modified Rosen T-16 (6) protocol, comprising ifosfamide $\left(2000 \mathrm{mg} / \mathrm{m}^{2} /\right.$ day, day $\left.1-5\right)$, etoposide $\left(120 \mathrm{mg} / \mathrm{m}^{2} /\right.$ day, day $\left.2-4\right)$, doxorubicin $\left(30 \mathrm{mg} / \mathrm{m}^{2} /\right.$ day, day 5 and 6) and cyclophosphamide $\left(1250 \mathrm{mg} / \mathrm{m}^{2} / \mathrm{day}\right.$, day 1 and 2) intravenously. A subsequent computed tomography scan revealed that the tumor had responded partially to the treatment as defined by the Response Evaluation Criteria in Solid Tumors (RECIST) criteria (7). Metastasectomies were then performed to remove multiple peritoneal metastases. Following these surgeries, the patient received five courses of chemotherapy, which followed the modified Rosen T-16 protocol, over a 5-month period. The patient then underwent three metastasectomies for isolated peritoneal metastases. In 2008, a follow-up computed tomography scan identified para-aortic lymph node metastases. The patient received radiation therapy for the recurrent nodal disease, and a computed tomography scan revealed a partial response by the tumor to this treatment as defined by RECIST criteria. However, despite treatment, the growth of the peritoneal metastases progressed and para-aortic and mediastinal lymph node metastases appeared. The patient succumbed due to progressive disease 110 months after the initial surgery for gastric ES/PNET.

\section{Discussion}

The term 'Ewing sarcoma' has been used for tumors that lack evidence of neuroectodermal differentiation as assessed by light microscopy, immunohistochemistry and electron microscopy, whereas the term 'PNET' has been employed for tumors that exhibit neuroectodermal features as evaluated by one or more of these modalities (8). Originally thought to be a separate entity, PNET is now known to commonly share 
similar immunohistochemical characteristics and an identical chromosomal translocation $\mathrm{t}(11 ; 22)(\mathrm{q} 24 ; \mathrm{q} 12)$ with ES. The two diseases are now treated as a single entity $(8,9)$. In the present case, the EWS-FLII fusion gene translocation $\mathrm{t}(11 ; 22)$ (q24;q12) indicated that the tumor arising from the muscularis externa layer of the stomach was a gastric ES/PNET.

The most useful immunohistologic reagent for the diagnosis of ES/PNETs is a monoclonal antibody to CD99 (HBA/71, 12E7 and 013) that is able to identifty a cell surface protein. This protein is the product of the MIC2 gene, which is located on the pseudoautosomal region of the $\mathrm{X}$ and $\mathrm{Y}$ chromosomes (1). Immunohistochemical staining using an antibody to CD99 has been strongly positive in 90 to nearly $100 \%$ of cases reported to be ES/PNETs $(10,11)$. An evaluation of the five reported cases of gastric ES/PNETs revealed that tumors from all cases showed positive immunoreactivity for CD99. Immunohistochemical staining for the intermediate filament vimentin is usually positive in ES/PNET cells (1). Similarly, the five reported cases of gastric ES/PNETs showed positive vimentin expression in tumor cells. Neural markers, such as S100, chromogranin A, synaptophysin and neuronspecific enolase, demonstrate variable immunohistochemical staining in ES/PNET cells, which may be due to the differing degrees of neuroectodermal differentiation in the individual tumors (1).

In the five reported cases of gastric ES/PNETs (Table I), surgical resection was possible in all cases. Although three of the reported cases, including our case, had distant metastases, only our patient underwent multiple repeat metastasectomies for recurrent disease. Since our patient survived more than 9 years following the initial surgery for gastric ES/PNETs, repeat metastasectomies for isolated recurrence, where feasible, may improve survival in patients with gastric ES/PNETs.

The current standard chemotherapeutical treatment for ES/PNETs is a regimen comprising the drugs: vincristine, doxorubicin, cyclophosphamide, ifosfamide and etoposide. Chemotherapy often enables the cure of ES/PNET patients with localized disease. However, in patients with metastatic spread its benefit is usually limited to extending the length of time of progression-free survival $(12,13)$. The findings of Haeusler et al demonstrated that combined modality treatment (surgery, multi-agent chemotherapy and radiotherapy) improved survival in patients with metastatic spread of ES/PNETs (14). The results of our case demonstrated that combined modality treatment was effective in achieving long-term survival. These results therefore indicate the importance of multimodal treatment approaches for patients with ES/PNETs.

In conclusion, gastric ES/PNETs are rare tumors with high metastatic potential. Multimodal treatment approaches including surgery, multi-agent chemotherapy and radiotherapy may provide a survival benefit for patients with gastric ES/ PNETs.

\section{References}

1. Kempson RL, Fletcher CDM, Evans HL, et al: Tumors of the Soft Tissues. 3rd edition. The Armed Forces Institute of Pathology, Washington, DC, pp444-452, 2001.

2. Czekalla R, Fuchs M, Stölzle A, et al: Peripheral primitive neuroectodermal tumor of the stomach in a 14-year-old boy: a case report. Eur J Gastroenterol Hepatol 16: 1391-1400, 2004. 
3. Soulard R, Claude V, Camparo P, Dufau JP, Saint-Blancard P and Gros P: Primitive neuroectodermal tumor of the stomach. Arch Pathol Lab Med 129: 107-110, 2005.

4. Rafailidis S, Ballas K, Psarras K, Pavlidis T, Symeonidis N, Marakis G and Sakadamis A: Primary Ewing sarcoma of the stomach - a newly described entity. Eur Surg Res 42: 17-20, 2009.

5. Colovic RB, Grubor NM, Micev MT, Matic SV, Atkinson HD and Latincic SM: Perigastric extraskeletal Ewing's sarcoma: a case report. World J Gastroenterol 15: 245-247, 2009.

6. Obata H, Ueda T, Kawai A, et al: Clinical outcome of patients with Ewing sarcoma family of tumors of bone in Japan: the Japanese Musculoskeletal Oncology Group cooperative study. Cancer 109: 767-775, 2007.

7. Therasse P, Arbuck SG, Eisenhauer EA, et al: New guidelines to evaluate the response to treatment in solid tumors. European Organization for Research and Treatment of Cancer, National Cancer Institute of the United States, National Cancer Institute of Canada. J Natl Cancer Inst 92: 205-216, 2000.

8. Ushigome S, Machinami R and Sorensen PH: Ewing sarcoma/ Primitive neuroectodermal tumour (PNET). In: World Health Organization Classification of Tumours. Pathology and Genetics of Tumours of Soft Tissue and Bone. Fletcher CDM, Unni KK and Mertens F (eds). IARC Press, Lyon, pp298-300, 2002.

9. Skubitz KM and D'Adamo DR: Sarcoma. Mayo Clin Proc 82: 1409-1432, 2007.
10. Ambros IM, Ambros PF, Strehl S, Kovar H, Gadner H and Salzer-Kuntschik M: MIC2 is a specific marker for Ewing's sarcoma and peripheral primitive neuroectodermal tumors. Evidence for a common histogenesis of Ewing's sarcoma and peripheral primitive neuroectodermal tumors from MIC2 expression and specific chromosome aberration. Cancer 67: 1886-1893, 1991.

11. Ramani P, Rampling D and Link M: Immunocytochemical study of 12E7 in small round-cell tumours of childhood: an assessment of its sensitivity and specificity. Histopathology 23 : 557-561, 1993.

12. Leavey PJ and Collier AB: Ewing sarcoma: prognostic criteria, outcomes and future treatment. Expert Rev Anticancer Ther 8: 617-624, 2008

13. Esiashvili N, Goodman M and Marcus RB Jr: Changes in incidence and survival of Ewing sarcoma patients over the past 3 decades: Surveillance Epidemiology and End Results data. J Pediatr Hematol Oncol 30: 425-430, 2008.

14. Haeusler J, Ranft A, Boelling T, et al: The value of local treatment in patients with primary, disseminated, multifocal Ewing sarcoma (PDMES). Cancer 116: 443-450, 2010. 